\title{
Nuevo horizonte de sentido histórico, crisis de la modernidad y movimientos de la sociedad. El legado teórico de Aníbal Quijano
}

Recibido: 16/02/2019

Aprobado:20/05/2019
JULIO MEJÍA NAVARRETE

Universidad Nacional Mayor de San Marcos

jvmenal@gmail.com

\section{RESUMEN}

En un periodo de surgimiento de pensamientos retrógrados y nostálgicos, de la cultura de la desesperanza, es fundamental analizar la propuesta teórica de Aníbal Quijano de la emergencia de un horizonte de sentido histórico, del desarrollo de otro imaginario de futuro sustentado en valores morales de justicia humana, resultado de las respuestas de transformación que se generan en las mismas fuerzas y movimientos de la sociedad.

Palabras clave: Horizonte de sentido histórico, modernidad, movimientos de la sociedad, dignidad.

\section{New horizon of historical sense, crisis of modernity and movements of the society. The theoretical legacy of Anibal Quijano}

\begin{abstract}
In a period, that gestates retrograde and nostalgic thoughts, in the culture of despair, it is fundamental to analyze the theoretical proposal of Aníbal Quijano on the emergence of a horizon of historical sense. In which the development of another imaginary of the future based on moral values of human justice, comes as result of the transformation responses generated in the same forces and movements of society.
\end{abstract}

Keywords: Horizon of historical sense, modernity, movements of the society, dignity. 
ctualmente, se viven tiempos donde prima la cultura de la desesperanza
que oprime al mundo, la crisis de la democracia liberal, los regímenes
"populistas" y de extrema derecha, las serias dificultades de la Unión
Europea, la pugna entre las potencias, el aumento exponencial de la polarización social y la pobreza, la expansión del racismo, sexismo y la devastación de la naturaleza que ponen en peligro la vida misma en el planeta. En ese contexto, es importante detenerse en la propuesta de Aníbal Quijano de la emergencia de un horizonte de sentido histórico, del desarrollo de otro imaginario de futuro de justicia de la humanidad, como resultado de la comprensión profunda de las respuestas de transformación que se generan en la mismas fuerzas y movimientos de la sociedad.

Por ello, el horizonte de sentido histórico es uno de los conceptos centrales en la perspectiva teórica de Aníbal Quijano. ${ }^{1}$ Significa pensar en un orden de igualdad social y de vida de la naturaleza, que incluye la revisión de los propios fundamentos de la hegemonía eurocéntrica de la dominación moderna. El horizonte de sentido histórico es un proceso que fluye desde el desasosiego e incertidumbre frente a los cambios críticos de la modernidad neoliberal, amenazas que golpean la ecología y la propia vida. Para hacerle frente suscitan una reacción intensa de indignación y descontento de los movimientos de la sociedad que reintroducen miradas disconformes para reorganizar la existencia en la Tierra y, a partir de ahí, propiciar un pensamiento que gire en función de la esperanza y la imaginación de otro orden social. El horizonte de sentido histórico es la vuelta a una creencia, idea e imaginario de la "búsqueda de otra sociedad, de otra historia" (Quijano, 2009, p. 34), portadora de ilusiones de un futuro diferente a la modernidad capitalista, del advenimiento de un tiempo nuevo de otra existencia de justicia social.

De esa forma, la modernidad en pleno cuestionamiento y metamorfosis puntea el advenimiento de comportamientos sociales que apuntalan la posibilidad de un nuevo horizonte de sentido histórico. Manuel Castells (2009, p. 207) describe formas de acción humana que se generan a partir de las respuestas frente a los temores de la moderna sociedad del riesgo, donde "El miedo es esencial para la autoconservación, pero la esperanza es fundamental para la supervivencia" de la humanidad, mediante las reacciones de indignación de los

1 Puede consultarse los siguientes trabajos de Aníbal Quijano: Modernidad, identidad y utopía en América latina (1988), "Estética de la utopía" (1990), "El regreso del futuro y las cuestiones del conocimiento" (2001), "El nuevo imaginario anticapitalista" (2002), "La crisis del horizonte de sentido colonial/moderno/ eurocentrado" (2009a) y "Otro horizonte de sentido" (2009b). 
movimientos societales que introducen nuevos valores y discursos libertarios, como formas pensamiento centrados en "la capacidad para imaginar el futuro [...] orientado a conseguir el bienestar futuro como consecuencia de una actuación presente".

Lo sustancial del horizonte de sentido histórico es el desarrollo de una cultura de la esperanza, de la igualdad social y de la vida en la naturaleza, cimentada en un ideal moral que facilite la crítica a la modernidad global, a los males de la vida actual, y que permita la desaprobación de los abusos y contraponga drásticamente la realidad existente con la aspiración moral de otra sociedad justa. Sobre todo, en una época de surgimiento de imaginarios retrógrados y nostálgicos, lo que Zygmunt Bauman (2017, p. 14) explicara tan lucidamente como "retropías, que son mundos ideales ubicados en un pasado [...] y no en ese futuro todavía por nacer".

El horizonte de sentido no manifiesta un modelo acabado, formula un principio ético que delimita una trayectoria de posibilidades futuras, alienta una oferta de sentido que motiva la acción individual y permite movilizar las fuerzas latentes de la sociedad. El ideal puede favorecer a desarrollar una sociedad más justa como un camino de práctica y experimentación del progreso humano, nunca como un proyecto terminado.

Este trabajo analiza la emergencia del horizonte de sentido histórico alternativo, siguiendo las propuestas teóricas de Aníbal Quijano. El estudio no espera ser acabado, lo esencial es subrayar algunos elementos que permitan establecer un debate mayor. Si bien interesa esbozar las ideas fundamentales del autor, el examen del horizonte de sentido histórico únicamente puede mostrarse en directa interconexión con "los referentes demostrativos, en consecuencia victoriosos, en la experiencia concreta" (Quijano 2001, p. 8).

En suma, el texto a partir del concepto horizonte de sentido histórico examina sus interrelaciones con la crisis de la modernidad y el desarrollo de los movimientos de la sociedad.

\section{Crisis raigal de la modernidad, desigualdad y destrucción ecológica}

La modernización global neoliberal a partir de la segunda mitad de la década de 1970 se expande en una dinámica de crecimiento constante, que viene produciendo cambios sistémicos en la sociedad. Wallerstein (2010) define a los primeros años del siglo XXI como un estado de fluctuaciones e incertidum- 
bres permanentes y Aníbal Quijano (2014a, p. 27) lo denomina “crisis raigal de la colonialidad global del poder". Pareciera que el sistema-mundo moderno, después de más de quinientos años, desde la conquista de América Latina, está llegando a sus límites. Destruye la naturaleza y delinea una concentración de la riqueza y las desigualdades sociales abismales que están produciendo un "monstruo climático" y un "monstruo histórico" que amenazan toda forma de vida (Quijano, 2014b, 12).

El crecimiento de la modernidad global se asocia con la dinámica de la nueva revolución tecnológica. La progresión acelerada del capital introduce un serio peligro sobre la Tierra, llevando a niveles catastróficos la brecha entre la acumulación ilimitada y los recursos definidos del planeta. Esto trae como resultado la crisis climática. En los Andes peruanos se han perdido irremediablemente el $42.64 \%$ de los glaciares de las cordilleras por el impacto del calentamiento global. Actualmente está en desarrollo la sexta extinción a gran escala de la vida en la Tierra; la ratio es hasta cien veces más alto que la tasa natural (Ceballos et al., 2015), y los últimos cuatro años han sido los de mayor temperatura de los que se tiene registro histórico. El equilibrio autopoiético de la Tierra se ha quebrado por la influencia depredadora de la modernización de los últimos cinco siglos, poniendo en serio riesgo toda forma de existencia en los próximos cien años. Se ha disparado la separación entre los intereses del orden moderno capitalista y la naturaleza.

Vinculado a lo anterior, la automatización y la inteligencia artificial en los procesos productivos y la reorganización del trabajo en la modernidad actual ya no genera masivamente trabajo asalariado. Lo paradójico es que ocurre cuando se produce más empleo que en cualquier otro momento de la historia (Standing, 2016). No obstante, el sistema moderno capitalista sigue reproduciéndose, lo hace recurriendo cada vez más a la precarización laboral, el desempleo estructural, la amplificación de la pequeña producción mercantil y formas que se acercan a relaciones esclavistas y serviles. El resultado de la modernización neoliberal es la brutal concentración de riquezas que llevan a la desigualdad social extrema (Piketty, 2015). En el Perú este proceso se manifiesta crudamente: el $70 \%$ de los trabajadores se encuentran en el sector informal. América Latina sigue siendo el continente de mayor disparidad social. En el mundo, el $10 \%$ de la población se apropiada del $88 \%$ de la riqueza y el $50 \%$ más pobre posee solo el $1 \%$ de la totalidad (Castells, 2015).

En general, la "crisis raigal" de la modernidad global traza un devenir trágico en la historia, no admite más concebir la esperanza de acabar con la 
miseria y el sufrimiento. Al contrario, la promesa moderna de justicia social ha sido burlada en América Latina y el mundo. Pareciera que se consolidad la expansión de la modernidad, pero sin los grandes logros que trazó originariamente; es decir, viene produciéndose una modernización sin precedentes del planeta, aunque sin las ambiciones libertarias de la modernidad (Mejía, 2016a).

\section{Nuevos discursos de los movimientos de la sociedad}

Los movimientos de la sociedad representan la lucha por la sobrevivencia frente a las amenazas de la extrema desigualdad y la destrucción de la naturaleza. Sin embargo, lo central es la emergencia de un pensamiento que se origina frente a las necesidades y en las ilusiones de un futuro de una sociedad fundada en la justicia social. En ese contexto, es la primera vez en los quinientos años de modernidad que emergen valores y un discurso de dignidad de cómo organizar en una unidad la vida social y la natural.

En las últimas décadas se ensayan diversas respuestas en América Latina y otras partes del mundo. En especial, luego de las acciones de organización y movilización de la población indígenas de la región de Chiapas en enero de 1994. Indígenas, afros, negros, etnicidades y grupos sociales diferenciados y subordinados cuestionan la idea de la raza, etnicidad, todas las formas de clasificación social y destrucción de la ecología. Las redes de "indignados" que se extendieron desde la Primavera Árabe en Europa, el movimiento "occupy" en Estados Unidos, el 15-M en España, los movimientos estudiantiles en Chile, \#Yosoy132 en México, la rebelión de los "pulpines" en Perú y las protestas contra la corrupción en Brasil instalan las demandas de igualdad social como la aspiración central frente al "1 \% más rico" de la sociedad. ${ }^{2}$ Asimismo, trabajadores, indios, negros, mestizos, mujeres, jóvenes, inmigrantes, homosexuales, pacifistas, antiutilitarios, antiglobalización y otros desarrollan un conjunto de idearios, demandas y medidas de lucha que aluden al reconocimiento de los derechos humanos, los derechos las mujeres, los derechos de los homosexuales, los derechos de la paz, solidaridad, reciprocidad, ecología y, en general, pugnan por la construcción de una sociedad más igualitaria y justa.

2 Puede consultarse Castells (2014, 2012), De Sousa Santos (2015), Zibechi (2015), Sandoval (2015), Rojas (2012), Aguilera (2016), Fernández-Maldonado (2015), González y Schneider (2016). 
Evidentemente, los movimientos indígenas en sus organizaciones y movilizaciones de la poblaciones y comunidades de la región acarrearon el cuestionamiento de todas las formas de racismo, discriminación y clasificación social para enarbolar los valores de igualdad de las personas y, a la vez, hicieron frente a la mercantilización de la ecología para enarbolar un discurso de amparo de las condiciones de sobrevivencia y defensa de la naturaleza (Quijano, 2006).

En los últimos cincuenta años, los movimientos de mujeres en América Latina han transformado la conciencia sobre sí mismas (Luna, 2004). Aunque el patriarcado se mantiene vigente, en forma individual y colectiva las mujeres se piensan como sujetos con imaginarios fundados en la igualdad social. Las relaciones patriarcales han derivado en situaciones paradójicas, pero se tiene claro que la autoridad masculina tiene que desistir, transformándose ellos mismos en su lado femenino. Se va apoderando del pensamiento de las gentes, las relaciones hombre y mujer en todos los espacios de la vida social se alteran y empiezan a rehacerse en función de valores de igualdad social y libertad.

Los movimientos estudiantiles y juveniles cuestionan las relaciones de dominio, explotación y procuran formas de igualdad social. Experiencias que exigen ser identificados como sujetos de dignidad, frente a la humillación y negación de sus derechos, siempre apuestan por sueños de un mundo de igualdad social. Pero lo más importante, comienzan a modificar radicalmente las relaciones de paternidad, tradicionalmente fundadas en la autoridad de origen biológica, por ser el padre quien engendra. Hoy la autoridad de la paternidad se ha convertido en un hecho moral: el hijo solo obedece y acepta la autoridad legítima del padre cuando viene acompañada de la confianza, el prestigio y la igualdad. La paternidad muestra el avance del valor y el discurso de la igualdad en la sociedad del siglo XXI (Mejía, 2016b).

Los movimientos de indignados que surcan el mundo, América Latina, el norte del África, Europa y los Estados Unidos, sitúan reclamos de igualdad como el anhelo principal frente a la polarización social del " 1 \% más rico" que se apropia del $99 \%$ de la riqueza global. Movimientos que vienen generando una modificación esencial en las mentalidades de las gentes sobre la injusticia de la exorbitante diferenciación social, la mayor de la historia humana, $\mathrm{y}$ forjan nuevos valores y discursos en torno a la dignidad humana, con un imaginario de igualdad y justicia social (Castells, 2012). 
Los movimientos ecologistas en su defensa del territorio y el medio ambiente bosquejan en América Latina un pensamiento que comprende lo humano como parte de la naturaleza, que lleva la recuperación de su animalidad, de constituir un ser vivo dentro de la infinita existencia heterogénea de la Tierra y del universo. Expresan un pensamiento que cuestiona la diferencia y superioridad humana, nos devuelve al mundo natural y define a todos como seres mortales e igualitarios. Los seres humanos tenemos que pensarnos como una especie más y común de la naturaleza para enfrentar los graves problemas que amenazan el planeta (Leff, 2004, pp. 124-185).

Los movimientos de inmigrantes se han transformado en una tendencia global involucrando a más diez millones de personas que desde el norte de África, Turquía y la frontera de México atraviesan principalmente Europa y Estados Unidos (Wieviorka, 2018, p. 341) demandando el acceso a la igualdad de oportunidades, seguridad, cobijo, empleo e igualdad de ciudadanía. Pero, sobre todo, son movimientos de inmigrantes, refugiados y minorías étnicas que se alzan contra el discurso xenófobo-racista y discriminador, muchas veces impulsado desde los propio Gobiernos, que busca cosificarlos, dominarlos e inferiorizarlos en seres subhumanos. Apegándose a lo último que les queda, invocan los valores de dignidad humana, de sueños de justicia social contra la destrucción y el sufrimiento.

El resultado de las prácticas de los movimientos de la sociedad es la emergencia de un nuevo imaginario que subraya los valores de igualdad social y de vida de la naturaleza. Se trata de un pensamiento que alumbra un mundo de esperanzas de organización radicalmente diferente de la existencia social.

\section{El emergente horizonte de sentido histórico: "Estamos comenzado a convivir con el futuro"}

Desde fines del siglo XX, en medio de la "crisis" de la modernidad globalizada y el desarrollo de los nuevos movimientos de la sociedad emerge un horizonte de sentido histórico. Se instituyen valores, ideas y una subjetividad que impugna el subterfugio de la naturalización de toda forma de diferencia social y afirman posibilidades de imaginar un mundo cimentado en la igualdad social y en la unidad de la sociedad con el universo.

En el contexto de cambios sistémicos, son los movimientos de la sociedad los que vuelven a instaurar valores culturales libertarios que cuestionan 
profundamente el patrón epistémico eurocéntrico. Así germina un nuevo discurso de horizonte de sentido histórico que empieza a desarrollar una nueva propuesta moral que expresa otro ideal del orden societal moderno, "una subversión del imaginario del mundo, una liberación de ese imaginario respecto de los patrones que lo estructuran y al mismo tiempo lo aprisionan” (Quijano, 1990, p. 34).

La particularidad de la emergencia del horizonte de sentido histórico fue explicada por Aníbal Quijano (2009b, p. 5): "No es solo el discurso, no son solo asambleas, están reorganizando sus comunidades, están asociando sus comunidades, están generando otra forma de autoridad política en el mundo que va a tener que competir y conflictuar con el Estado mientras el Estado aún esté allí. Estamos con otro elemento nuevo. Esto no es solo una utopía, eso está comenzando; estamos comenzando a convivir con el futuro. Podemos ser derrotados, incluso el mundo puede terminarse; pero no tiene vuelta atrás". Ahora se interrelacionan el discurso de la esperanza y la aparición germinal de relaciones y prácticas sociales.

De esa forma, el discurso del horizonte de sentido histórico congrega valores e ideales morales desarrollados por los movimientos de la sociedad en su lucha contra los patrones de explotación y dominación de la naturaleza y del hombre. Por una parte, el movimiento indígena y el movimiento ecológico "están defendiendo las últimas condiciones de existencia, sus últimas condiciones de sobrevivencia en el mundo. No pueden vivir más sin esa floresta, sin el oxígeno, sin el agua, sin los materiales que les permiten producir bienes culturales para su existencia. Esta es la primera vez que emerge otra propuesta de sentido histórico" (Quijano, 2009b, p. 5). Por otra parte, los movimientos de la sociedad son la fuerza central de la transformación contemporánea y abren un momento histórico de inversión colosal de la cultura, pierde legitimidad el viejo constructo mental sobre las desigualdades racial/étnica, de género, clase y de exclusiones, gana importancia formas categoriales originales que facilitan el pensar y organizar históricamente el orden presente del mundo en una cultura de la igualdad social (Bauman, 2014, pp. 83-91).

Las poblaciones dominadas por la modernidad globalizada emprenden un nuevo horizonte epistémico alternativo al eurocéntrico, asumen la conciencia de pertenecer a un solo pueblo: la humanidad, cuyo principio esencial es la dignidad de los seres humanos: "La humanidad actual en su conjunto constituye el primer sistema-mundo global históricamente" (Quijano, 2000, p. 214). 
Y la humanidad es el destino futuro, porque somos "múltiples culturas, [pero] una sola humanidad" (Bauman, 2008, p. 13). Las diferencias están en la historia y la heterogeneidad que habita un único mundo, el planeta Tierra.

El horizonte de sentido histórico en América Latina y en el mundo han introducido una etapa de modificaciones del pensamiento, la vieja "cultura de la dominación" eurocéntrica se encuentra cuestionada por "el nacimiento de la cultura" de la dignidad humana. Emerge un pensamiento que contradice la hegemonía de lo eurocéntrico, contribuyen desde caminos complejos diversas fuerzas, movimientos de la sociedad, núcleos de científicos, líderes sociales y la opinión pública internacional.

En efecto, a partir de la década de 1970 se viene desarrollando inéditas relaciones entre la comunidad científica y sectores importantes de personalidades que están generando una opinión pública internacional más sensible por los temas de la destrucción ecológica y la desigualdad en el mundo. En esas condiciones, se subrayan los trabajos de Thomas Piketty (2015) y de James Lovelock (2007), que están juntándose con partes importantes de la inteligencia contemporánea para insistir en denunciar la destrucción de las condiciones de vida en el planeta y la expansión de la polarización social. La preocupación sobre el calentamiento global y la desigualdad extrema han ido incrementándose entre los científicos expertos, interesados en el análisis para descifrar los resortes de la explotación y dominación del mundo contemporáneo, del "monstruo histórico" y el "monstruo climático" como las mayores amenazas de la modernidad global y comprometiendo a diversas personalidades del mundo, entre los que destacó las campañas de Al Gore frente al cambio climático (Castells, 2009, pp. 421-432).

En América Latina y el mundo actual se recrean valores de igualdad social y defensa de la naturaleza que posibilitan la irrupción de otra episteme, traduciéndose en el desarrollo de una opinión pública internacional. En primer lugar, Manuel Castells destaca "el nacimiento de la cultura ecológica" (2009, p. 408) en la mayoría de países, fundada en la preocupación por los temas medioambientales y por sus posibles efectos globales. En segundo lugar, se bosqueja un inédito proceso cultural modelado por su encuentro con las ideas de igualdad social, Bauman (2014, p. 103) lo expresa claramente: "Si preguntáramos a la gente por los valores más importantes para ellos, es muy probable que muchos contesten nombrando la igualdad”. En general, pareciera que asistimos a una transformación profunda y a la génesis de una nueva cultura mundial frente a las amenazas de la crisis ecológica y la desigualdad extrema. 
Los movimientos de la sociedad y los mayores niveles de sensibilidad en la comunidad científica y la germinación de una cultura libertaria inciden directamente en la formación de experiencias inéditas de representación, de autonomía y de autoridad alternativas en las múltiples redes asociativas (Cardoso et al., 2018, pp. 607-608). Se produce un giro esencial en la vida social que se desplaza a la gestación de "la cultura de la autonomía. La matriz fundamental de las sociedades contemporáneas" (Castells, 2012, p. 219), de revitalización comunal que tiene como punto de partida y su empoderamiento principal en las redes sociales, conjugando los intereses de las personas con las experiencias colectivas. Las redes sociales son precursoras de otros caminos de organización de la vida individual y societal, allí aparece la capacidad para transformase en sujeto "afirmando su autonomía respecto a las instituciones de la sociedad" (Castells, 2012, p. 220). Los sujetos se integran en las redes de comunidades desbordando los marcos de la sociedad, rechazan las instituciones existentes y buscan un nuevo contrato social que posibilite compatibilizar libertad individual con igualdad.

En particular, la autonomía de las redes es el potencial que posibilita a los individuos convertirse en sujetos con prácticas diferenciadas de las instituciones vigentes, ensayando conductas inéditas que van desde un espíritu innovador en el trabajo, consumidores enterados y proactivos en el mercado, alumnos informados y críticos en la educación, personas que pueden formular la atención centrada en el paciente de la sanidad, demandar audiencias activas en los medios de comunicación, propiciar el cambio cultural en los movimientos sociales, favorecer una ciudadanía informada, independiente y participativa en el Gobierno y la política (Castells, 2014). Asimismo, la progresión de la autonomía de las redes genera formas iniciales de organizaciones comunitarias fundadas en impulsos "democráticos igualitarios" que puedan ir más allá de la sociedad moderna, creando verdaderos "espacios de la autonomía", en donde destacan las experiencias concretas de las "utopías reales" (Wright, 2014), las sociedades "de coste marginal cero" (Ryfkin, 2014), las "prácticas económicas alternativas" generadas como respuestas populares a la "crisis que estalló en el 2008, primero en Estados Unidos y luego en Europa” (Castells, 2017, p. 294) y la expansión de "los bienes comunes del conocimiento" (Hess y Ostrom, 2016). En América Latina los esfuerzos de indígenas, negros y mestizos en las comunidades devienen en espacios autónomos "integrales", porque abordan todos los aspectos de la vida de las personas, desde las ideas, la producción, los alimentos, la salud hasta la justicia y el poder, en donde 
sobresalen las juntas del Buen Gobierno Zapatista, los cabildos de nasa del Cauca, las experiencias mapuches (Zibechi, 2015, p. 117). La pluralidad de experiencias en las redes de comunidad están condicionando una existencia social que pueda desenvolverse híbridamente dentro de la sociedad y fuera de ella pero, sobre todo, anunciando la ocurrencia de formas alternativas de autoridad como caminos de autonomía y transformación de las instituciones de la sociedad.

En general, los movimientos sociales, los importantes niveles de conciencia en la comunidad científica, la germinación de una cultura libertaria y las posibilidades de autonomía de las redes asociativas descubren históricamente la emergencia de un horizonte de sentido histórico de un mundo de justicia.

\section{Notas finales}

El artículo examina el desarrollo de un nuevo discurso del horizonte de sentido histórico a partir de la experiencia manifiesta de los movimientos de la sociedad en América Latina y el mundo contemporáneo. La modernidad global suscita un tiempo histórico de cambio epistémico que confronta la hegemonía del pensamiento eurocéntrico. Episteme que expresa las profundas modificaciones históricas del modo de pensar moderno que pretende imaginar otro mundo alternativo de libertad e igualdad social, de una nueva "racionalidad no-eurocéntrica, que pueda también ser parte del propio horizonte de futuro" (Quijano 2001, p. 17).

En un momento de grandes cambios y movimientos de la sociedad, el emergente horizonte de sentido histórico introduce un proceso de recuperación de la razón histórica fundada en una "ética de la responsabilidad" frente a la naturaleza y al hombre, de afirmación de la vida. Se abre un camino de transformación de la racionalidad medio-fin mercantil que conduce a la muerte en el mundo por la gestación de una nueva racionalidad basada en la supremacía de los fines del compromiso con la humanidad y la naturaleza (Hinkelammert y Mora, 2005).

Esta razón histórica retoma el sentido epistémico de la naturaleza como sujeto y del hombre como "sujeto vivo", que se opone radicalmente a la memoria de la modernidad/colonialidad de considerarlos como meros objetos externalizados, cosificados y desechables. Los movimientos de la sociedad en su defensa del territorio y la ecología del mundo bosquejan un imaginario de 
la naturaleza como ser vivo (Leff, 2004, pp. 124-185), no solo como fuente de toda forma de vida sino, principalmente, como sujeto de vida, que se autoproduce en un proceso histórico de interrelación entre lo químico, biológico y físico, sujeto con derechos plenos, perspectiva que rescata la vieja idea de las culturas andinas de la pachamama como madre naturaleza. Por otro lado, más allá de la visión cartesiana de lo humano como ser barbarizado, indigenizado y cosificado para la explotación del capital y la dominación racializada, los movimientos de la sociedad evocan la idea de "sujeto vivo", del ser yo persona (Hinkelammert, 2002) y con "autonomía cultural y personal" (Castells, 2009, pp. 471-473) frente a las instituciones sociales, con derechos reconocidos y con igualdad de acceso a los recursos que posibiliten el desarrollo de una vida integral, de igualdad social de individuos diversos y libres. "Estas prácticas representaban los embriones de una nueva economía (y sociedad), surgidos de la necesidad de sustituir al capitalismo disfuncional, pero también estaban motivadas por la búsqueda de una vida con sentido" (Castells y Hlebik, 2017, p. 257), de un horizonte posible.

Precisamente, el original discurso en América Latina es parte del proceso franqueado por la modernidad global de un tiempo histórico de bifurcación y emergencia de otros pensamientos. Supone destacar su persistencia cultural, porque fue el campo mental fundante y conflictivo permanente con la modernidad y colonialidad, desde sus orígenes con Guamán Poma, seguido por José Carlos Mariátegui, hasta las propuestas actuales del buen o bien vivir que trazan un camino de búsqueda de un orden definido por valores de justicia entre los seres humanos y la sociedad con la naturaleza (Mejía, 2016a). La gestación de un horizonte de sentido histórico en América Latina es la permanente pugna por "desprenderse" de la hegemonía del universalismo eurocéntrico abstracto y forma parte de todas las experiencias y prácticas de los colonizados del mundo que intentan confluir en un universalismo concreto depositario de todos los particulares, como lo anhelaba Aimé Césaire (2016).

En general, en un mundo en descomposición, el aporte teórico de Aníbal Quijano no se detiene en las promesas nostálgicas y reaccionarias, sino que busca identificar valores y discursos éticos en los nuevos movimientos de la sociedad para descubrir posibles caminos futuros de una sociedad justa, igualitaria y de vida de la naturaleza. 


\section{Bibliografía}

Aguilera, O. (2016). Movidas, movilizaciones y movimientos. Cultura política y políticas de las culturas juveniles en el Chile de hoy. Santiago de Chile: Ril Editores.

Bauman, Z. (2017). Retropía. Barcelona: Paidós.

Bauman, Z. (2014). ¿La riqueza de unos pocos no beneficia a todos? Bogotá: Paidós.

Bauman, Z. (2008). Múltiples culturas, una sola humanidad. Barcelona: Katz, CCCB.

Cardoso, G.; Accornero, G.; Lapa, T. y Azevedo, J. (2018). Movimientos sociales, participación y crisis en Europa. En Castells, M. (Ed.). Las crisis de Europa. Madrid: Alianza Editorial.

Castells, M. y Hlebik, S. (2017). Otra economía es posible. Cultura y economía en tiempos de crisis. Madrid: Alianza Editorial.

Castells, M. (2015). Sociedades polarizadas. La Vanguardia (17 de octubre): Barcelona. Recuperado de: https://www.lavanguardia.com/opinion/articulos/20151017/544-37274849/sociedades-polarizadas-manuel-castells.html.

Castells, M. (2014). El impacto de internet en la sociedad: una perspectiva global. California: BBVA, Open Mind.

Castells, M. (2012). Redes de indignación y esperanza. Los movimientos sociales en la era de internet. Madrid: Alianza Editorial.

Castells, M. (2009). Comunicación y poder. Madrid: Alianza Editorial.

Ceballos, G.; Ehrlich, P. R.; Barnosky, A.; Andrés García, A.; Pringle, R. M. y Palmer, T. M. (2015). Accelerated Modern Human-Induced Species Losses: Entering the Sixth Mass Extinction. Science Advances, 1(5). Recuperado de: http://advan-ces.sciencemag.org/content/advances/1/5/ e1400253.full.pdf.

Césaire, A. (2016). Discurso sobre el colonialismo. Madrid: Akal.

De Sousa Santos, B. (2015). Construyendo la contrahegemonía: traducción intercultural entre los movimientos sociales. Sandoval, R. (Ed.). Pensar desde la resistencia anticapitalista y la autonomía. México D. F.: CIESAS.

Fernández-Maldonado, E. (2015). La rebelión de los pulpines. Jóvenes, trabajo y politica. Lima: Otra Mirada.

González, R. y Schneider, A. (Comps.) (2016). Sociedades en conflicto. Movimientos sociales y movimientos armados en América Latina. Buenos Aires: CLACSO.

Hess, C. H. y Ostrom, E. (2016). Los bienes comunes del conocimiento. Madrid: IAEN, Traficantes de Sueños.

Hinkelammert, F. (2002). Crítica de la razón utópica. Bilbao: Desclée de Brouwer.

Hinkelammert, F. y Mora, H. (2005). Hacia una economía para la vida. San José: DEI. 
Leff, E. (2004). Racionalidad ambiental. La reapropiación social de la naturaleza. México D. F.: Siglo XXI Editores.

Lovelock, J. (2007). La venganza de la tierra. La teoría de la Gaia y el futuro de la humanidad. Barcelona: Editorial Planeta.

Luna, L. (2004). Los movimientos de mujeres en América Latina y la renovación de la historia. México D. F.: Fem-e-libros.

Mejía, J. (2016a). América Latina, modernidad y conocimiento. El desarrollo de otro discurso epistémico. Lima: Fondo Editorial de la Facultad de Ciencias Sociales de la Universidad Nacional Mayor de San Marcos.

Mejía, J. (2016b). Cultura y dignidad en América Latina. Descolonialidad de la desigualdad social. Investigaciones Sociales, 37, 315-324.

Piketty, T. (2015). El capital del siglo XXI. México D. F.: Fondo de Cultura Económica, Quijano, A. (2015). Notas sobre la descolonialidad del poder. Yuyaykusun, 8, 15-30.

Quijano, A. (2014a). Bien vivir: entre el "desarrollo" y la des/colonialidad del poder. En Quijano, A. (Ed.). Des/colonialidad y bien vivir. Un nuevo debate en América Latina. Lima: Universidad Ricardo Palma.

Quijano, A. (2014b). Presentación. En Quijano, A. (Ed.). Des/colonialidad y bien vivir. Un nuevo debate en América Latina. Lima: Universidad Ricardo Palma.

Quijano, A. (2009a). La crisis del horizonte de sentido colonial/moderno/eurocentrado. En Mejía, J. (Ed.). Sociedad, cultura y cambio en América Latina. Lima: Universidad Ricardo Palma.

Quijano, A. (2009b). Otro horizonte de sentido. América Latina en Movimiento, 441, 2-5.

Quijano, A. (2006). El "movimiento indígena" y las cuestiones pendientes en América Latina. Review Fernand Braudel Center, XXIX(2), 189-218.

Quijano, A. (2002). El nuevo imaginario anticapitalista. América Latina en Movimiento, $351,14-21$.

Quijano, A. (2001). El regreso del futuro y las cuestiones del conocimiento. Hueso Húmero, 38, 3-17.

Quijano, A. (2000). Colonialidad del poder, eurocentrismo y América Latina. En Lander, E. (Ed.). La colonialidad del saber: eurocentrismo y ciencias sociales. Perspectivas latinoamericanas. Buenos Aires: CLACSO, UNESCO.

Quijano, A. (1990). Estética de la utopía. Hueso Húmero, 27, 32-42.

Quijano A. (1988). Modernidad, identidad y utopía en América latina. Lima: Sociedad y Política Ediciones.

Rojas, J. (2012). Sociedad bloqueada. Movimiento estudiantil, desigualdad y despertar de la sociedad chilena. Santiago de Chile: Ril Editores. 
Ryfkin, J. (2014). La sociedad de coste marginal cero. El internet de las cosas y el eclipse del capitalismo. Barcelona: Paidós.

Sandoval, R. (Ed.) (2015). Pensar desde la resistencia anticapitalista y la autonomía. México D. F.: CIESAS.

Standing, G. (2016). El precariado y la lucha de clases. Sin Permiso: República y Socialismo También para el Siglo XXI, 15, 87-102.

Wallerstein, 1. (2010). Latinoamérica y los movimientos antisistémicos. En Negri, T.; Dussel, E.; Spivak, G. et al. I Ciclo de Seminarios Internacionales. Pensando desde Bolivia. La Paz: Vicepresidencia del Estado Plurinacional de Bolivia, Presidencia de la Asamblea Legislativa Plurinacional.

Wieviorka, M. (2018). Europa frente al mal: xenofobia, racismo, antisimetismo y terrorismo. En Castells, M. (Ed.). Las crisis de Europa. Madrid: Alianza Editorial.

Wright, E. O. (2014). Construyendo utopias reales. Madrid: Akal.

Zibechi, R. (2015). Descolonizar el pensamiento crítico y las prácticas emancipatorias. Bogotá: Ediciones desde Abajo. 